\title{
Mirko D'Onofrio, Paola Capelli and Paolo Pederzoli (Eds) Imaging and Pathology of Pancreatic Neoplasms. A Pictorial Atlas
}

\author{
Springer-Verlag Italia, 2015 ISBN 978-88-470-5677-0
}

\author{
Giuseppe Danilo Di Stasio $^{1} \cdot$ Luigi Mansi $^{1}$
}

Published online: 19 March 2016

(C) Springer-Verlag Berlin Heidelberg 2016

The editors of this book are Mirko D'Onofrio and Paola Capelli, who are members, repectively, of the Radiology and Pathology Departments at the G.B. Rossi University Hospital in Verona, and Paolo Pederzoli, a surgeon working in the "Casa di cura Pederzoli" in Peschiera del Garda (Verona). All of them are members of the famous "Verona's Pancreas Centre", the most important multi-specialized organization in Italy fully dedicated to pancreas diseases, with a vast amount of experience based on more than 40 years of tradition, steadily updated. Since the book has been written by a large series of experts, the contribution of Alec J. Megibow has been particularly evidenced on the cover.

This pictorial Atlas is structured into ten chapters, for a total of 432 pages, that cover the entire spectrum of pancreatic neoplasms. The dissertation starts with the most common pancreatic tumor, the ductal adenocarcinoma, and continues by analyzing all major and minor variants, including among others intraductal papillary mucinous neoplasms and descriptions of rare forms, such as neuroendocrine and cystic tumors. The last two chapters represent an appropriate conclusion of such a good work, as they explore "Driving Questions and Flowcharts" and the review of "Imaging Stigmata", providing a handy guide of interpretation for

Luigi Mansi

luigi.mansi@unina2.it

1 Second University of Naples, Napoli, Italy any clinical situation. As any good Atlas should be, the accent has been placed on images rather than the text itself, which instead is condensed at the beginning of each chapter. The pathological part is highlighted in yellow, and divided into two sections, macroscopy and microscopy, whereas the imaging section is arranged per technique, including Ultrasounds (US), Computed Tomography (CT) and Magnetic Resonance Imaging (MRI), thus providing an organized view on every aspect. After a concise evaluation of clinical presentation, behavior and major variants, the conclusion is entrusted to three crucial paragraphs that analyze "Pitfalls and Errors", "Imaging-Pathologic Correlation" and "Differential Diagnosis", thus helping the reader to unravel any doubts. In each chapter, at the end of the text part, the "Image Gallery" begins, a substantial collection of highquality images, both pathological, including surgical specimens and histopathology, and radiological, including US, $\mathrm{CT}$ and MRI, all of which are supplied with direct captions that guide the reader.

This Atlas represents the result of Professor Carlo Procacci's idea, which was revived in 2012 by Paola Capelli, as stated in the inscription by Mirko D'Onofrio himself. Thanks to the contributions of some of the most important national and international experts in the field of pancreatic neoplasms, it grants a unique focus on both pathological and radiological aspects, thus providing an efficient complementary vision to radiologists and pathologists respectively, and a complete guide to interpretation to both students and residents. Of course, this publication has to find its position in each medical library, as reference book for all the people who want to learn or remember the most important issues concerning imaging and pathology of pancreatic neoplasms. 